\title{
Analyzing the total structural intensity in beams using a homodyne laser doppler vibrometer $^{1}$
}

\author{
Agnaldo A. Freschi ${ }^{\mathrm{a}}$, Allan K.A. Pereira ${ }^{\mathrm{a}}$, \\ Khaled M. Ahmida ${ }^{\mathrm{a}}$, Jaime Frejlich ${ }^{\mathrm{b}}$ and \\ José Roberto F. Arruda ${ }^{a}$ \\ ${ }^{a}$ Departamento de Mecanica Computacional, FEM, \\ Universidade Estadual de Campinas C.P. 6122, \\ Campinas, SP, 13083-970 Brazil \\ ${ }^{\mathrm{b}}$ Laboratório de Óptica, IFGW, Universidade \\ Estadual de Campinas, Brazil
}

\section{Received 11 June 1999}

Revised 26 June 2000

The total structural intensity in beams can be considered as composed of three types of waves: bending, longitudinal, and torsional. In passive and active control applications, it is useful to separate each of these components in order to evaluate their contribution to the total structural power flowing through the beam. In this paper, a twisted $z$-shaped beam is used in order to allow the three types of waves to propagate. The contributions of the structural intensity, due to these waves, are computed from measurements taken over the surface of the beam with a simple homodyne interferometric laser vibrometer. The optical sensor incorporates some polarizing optics, additional to a Michelson type interferometer, to generate two optical signals in quadrature, which are processed to display velocities and/or displacements. This optical processing scheme is used to remove the directional ambiguity from the velocity measurement and allows nearly all backscattered light collected from the object to be detect. This paper investigates the performance of the laser vibrometer in the estimation of the different wave components. The results are validated by comparing the total structural intensity computed from the laser measurements, with the measured input power. Results computed from measurements using PVDF sensors are also shown, and compared with the non-intrusive laser measurements.

Keywords: Structural power flow, vibration intensity, laser vibrometers, wave components, wave propagation

\section{Introduction}

Predicting and measuring elastic waves, propagating through a structure, can be of foremost importance in vibroacoustic problems. The prediction and the measurement of the propagating elastic waves within a structure is usually referred to as structural power flow or structural intensity. The energy flow to localized dampers or to neighboring structures and supports can be cardinal mechanisms through which structural vibration is damped out, which partly explains the practical difficulty in estimating internal damping coefficients in structures using ground vibration tests. It can also be a key for solving structure-borne noise problems, by channeling vibrations to where they do not radiate noise, instead of trying to suppress them.

Structural intensity refers to the active part of vibration energy. As active energy is usually only a small fraction of the total vibration energy, estimating it from measured vibration is not always a simple task. Measuring structural intensity is more elaborate than measuring acoustic intensity. Sound propagates through air in compression-type waves only, while vibration propagates through solids in two basic types of waves: compression and shear waves. Depending on the geometry of the continuum, these two basic types of waves combine into different types of waves, such as bending, torsional, and longitudinal waves, which must all be measured.

Both acoustic intensity and vibration intensity measurements are associated with cross measurements between closely-spaced transducers: microphones in the

\footnotetext{
${ }^{1}$ An earlier form of this paper was presented at the 3rd International Conference on Vibration Measurements by Laser Techniques, Ancona, Italy, June 1998. The conference was organized by the Italian Association of Laser Velocimetry. The articles were selected, edited, and reviewed by a committee chaired by: Professor Enrico Primo Tomasini, Depertment of Mechanics, Faculty of Engineering University of Ancona, Italy.
} 


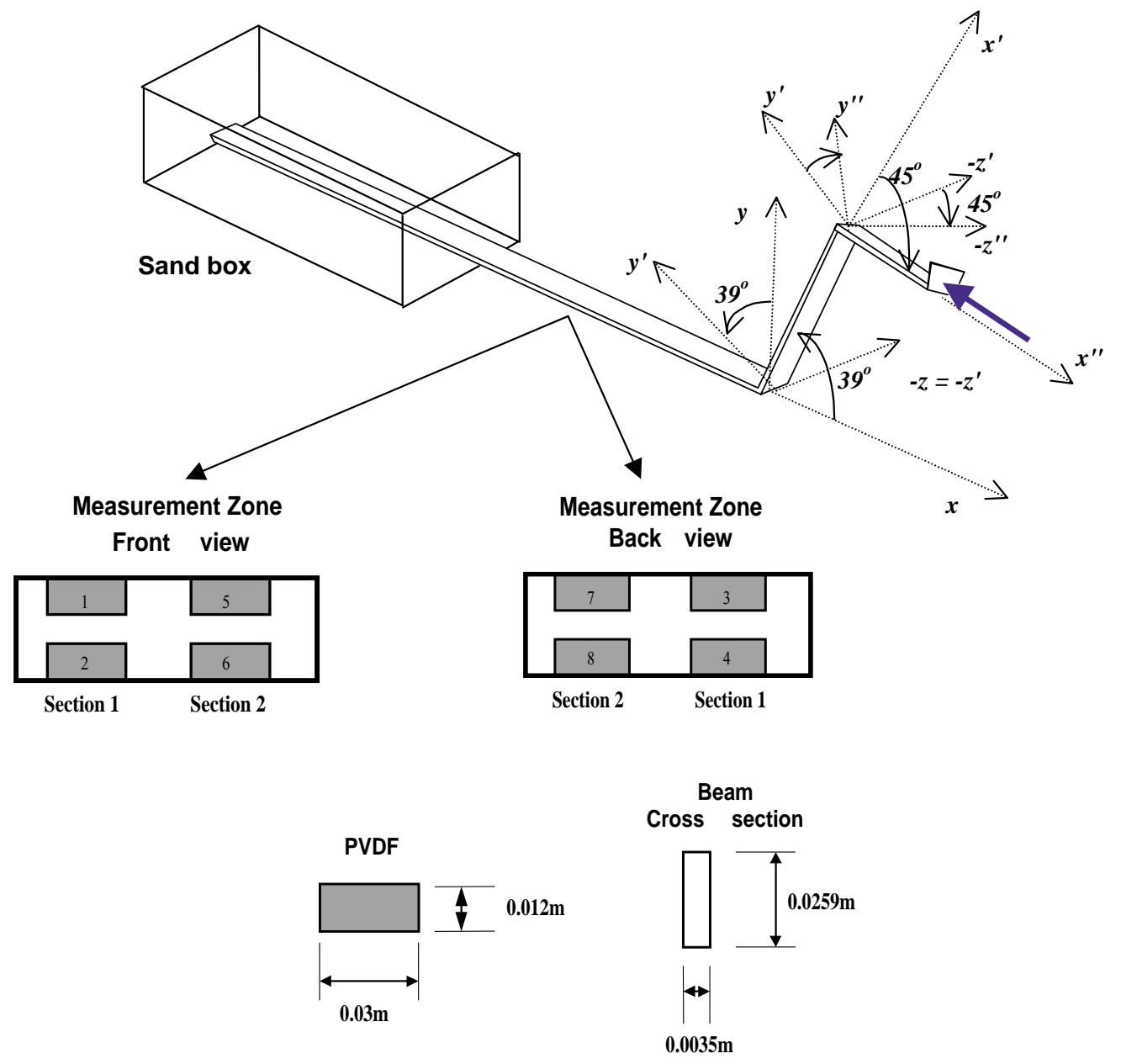

Fig. 1. $Z$-shaped beam with a quasi-anechoic termination (sand box).

former case and, usually, accelerometers or strain sensors in the latter. Following the original work by Noiseux [10], many authors have investigated different ways of computing the bending vibration intensity from measured accelerations in beams and plates $[1,11$, 13]. Most authors use finite-difference approximations to compute the partial spatial derivatives that are necessary to compute the power flow, but the wave component approach [6] is more general and does not require closely spaced measurements.

In this paper, a $z$-shaped beam (see Fig. 1), where the three kinds of waves - bending, longitudinal, and torsional - propagate, is used to investigate the difficulties in estimating the total structural intensity. The contributions of the structural intensity, due to these kinds of waves, are computed from measurements taken over the surface of the beam, with a simple homodyne interferometric laser vibrometer and with piezoelectric film sensor patches (PVDF). The optical sensor, which was developed in our laboratories, is briefly described.

\section{Structural intensity components}

The total structural intensity (SI) depends on the kinds of forces and moments that excite it. Each component of these forces and moments is associated with a different kind of wave propagating through the structure. In general, the total structural intensity, in a straight beam segment, along the $x$-axis can be considered to be composed of the following kinds of waves: a bending wave in the $x y$-plane, a bending wave in the $x z$-plane, a longitudinal wave in the $x$ direction and a torsional wave (angle $\theta$ in the $y z$-plane). In vibration control applications, it can be useful to find a way to separate these components, in order to calculate the 
total SI. Berthelot et al. [3] used a Laser Doppler Vibrometer (LDV) to separate the contributions from the structural intensity carried by the longitudinal waves in the presence of strong flexural waves.

Due to the geometry of the cross section of the $z$ shaped beam and due to the low frequency range that was investigated, bending in the $x z$-plane was assumed negligible (the beam is much stiffer in this plane). In this configuration all three of the kinds of waves mentioned propagate in the last segment of the beam (the measurement zone). The purpose here is therefore to separate the contributions of the SI due to the three kinds of waves using an LDV and compare the results obtained with those obtained using PVDF patches.

First, consider the bending waves using BernoulliEuler's theory, which is suitable for thin beams at low frequencies. The bending SI component may be expressed as [10]

$$
P_{b}=E I\left\langle\frac{\partial^{3} w}{\partial x^{3}} \frac{\partial w}{\partial t}-\frac{\partial^{2} w}{\partial x^{2}} \frac{\partial^{2} w}{\partial x \partial t}\right\rangle_{t}
$$

where $E$ is Young's modulus, $I$ is the inertia moment of the cross section of the beam, \langle\rangle$_{t}$ denotes the time average, and $w$ is the displacement in the $y$ direction. Considering only the far field components and using PVDF sensors of length $2 \mathrm{~d}$ spaced by $\Delta x$, bonded to the surface of the beam, it is possible to show that [6]

$$
P_{b f f}=\frac{2 E I \omega}{t^{2} k_{b} r_{b}^{2} \sin \left(k_{b} \Delta x\right)} \Im\left\{\varepsilon_{1}^{*} \varepsilon_{2}\right\}
$$

where $r_{b}=\frac{\sin \left(k_{b} d\right)}{k_{b} d}, k_{b}^{4}=\omega^{2} \frac{S \rho}{E I}$ is the flexural wavenumber, $\rho$ is the density, $S$ is the cross-section area, $t$ is half of the beam thickness, $\varepsilon_{i}$ is the complex amplitude of the strain measured at position $i$, $\Im$ is the imaginary part of a complex function, and argument $\omega$ is suppressed for clarity. The PVDF sensors are used to measure the surface strain.

The SI component, due to longitudinal waves, can be written as [4]

$$
P_{L}=-S E\left\langle\frac{\partial u}{\partial x} \frac{\partial u}{\partial t}\right\rangle_{t}
$$

where $u$ is the displacement in the $x$ direction.

Again, using PVDF sensors, it is possible to show that

$$
P_{L}=\frac{S E \omega}{2 k_{L} r_{L}^{2} \sin \left(k_{L} \Delta x\right)} \Im\left\{\varepsilon_{1}^{*} \varepsilon_{2}\right\}
$$

where $r_{L}=\frac{\sin \left(k_{L} d\right)}{k_{L} d}$ and $k_{L}^{2}=\omega^{2} \frac{\rho}{E}$ is the longitudinal wavenumber.

When using the LDV, both out-of-plane and in-plane velocities are measured. Therefore, we will now review the expressions for the structural intensity in beams using velocities. The flexural SI component can be written as [10]

$$
P_{b f f}=\frac{2 E I k_{b}^{3}}{\omega \sin \left(k_{b} \Delta x\right)} \Im\left\{\dot{W}_{1}^{*} \dot{W}_{2}\right\}
$$

where $\dot{W}$ is the complex amplitude of the transverse velocity component associated with bending.

The longitudinal SI component can be written as [10]

$$
P_{L}=\frac{S E k_{L}}{2 \omega \sin \left(k_{L} \Delta x\right)} \Im\left\{\dot{U}_{1}^{*} \dot{U}_{2}\right\}
$$

where $\dot{U}$ is the complex amplitude of the longitudinal velocity. The correction in the velocity, proposed by Berthelot et al. [3], was used in order allow for the measured longitudinal velocity component induced by the flexural waves.

Finally, the torsional SI component may be expressed as [4]

$$
P_{T}=-T\left\langle\frac{\partial \theta}{\partial x} \frac{\partial \theta}{\partial t}\right\rangle_{t}
$$

where $T=G b h^{3} / 3$ is the torsional stiffness, $b$ is the width of the beam, and $G$ is the shear modulus. Using the LDV measurements

$$
P_{T}=\frac{T \omega k_{T}}{2 b^{2} \omega \sin \left(k_{T} \Delta x\right)} \Im\left\{\dot{V}_{1}^{*} \dot{V}_{2}\right\}
$$

where $k_{T}^{2}=\omega^{2} \frac{b^{2} \rho}{16 h^{2} G}$ and $\dot{V}$ is the complex amplitude of the rotational velocity.

The input power was computed using the expression:

$$
P_{\text {in }}=\frac{1}{2} \Re\left\{F \dot{U}^{*}\right\}=\frac{1}{2 \omega} \Im\left\{F \ddot{U}^{*}\right\}
$$

where $F$ is the excitation force, $\dot{U}$ is the velocity, and $\ddot{U}$ is the acceleration in the direction of the applied force. Notice that in the above expressions, the complex real and imaginary components are in phase and in quadrature with the input force, respectively.

\section{The homodyne laser Doppler vibrometer}

Laser Doppler Vibrometers are instruments used to measure velocities and/or displacements of points on a vibrating surface by focusing a laser beam onto the object. The operation principle of all LDVs relies on the detection of the Doppler shift in the frequency of the light back-scattered by the surface. In most applications, the Doppler shifts are too low to be measured by a spectrometer, so the light collected from the object 


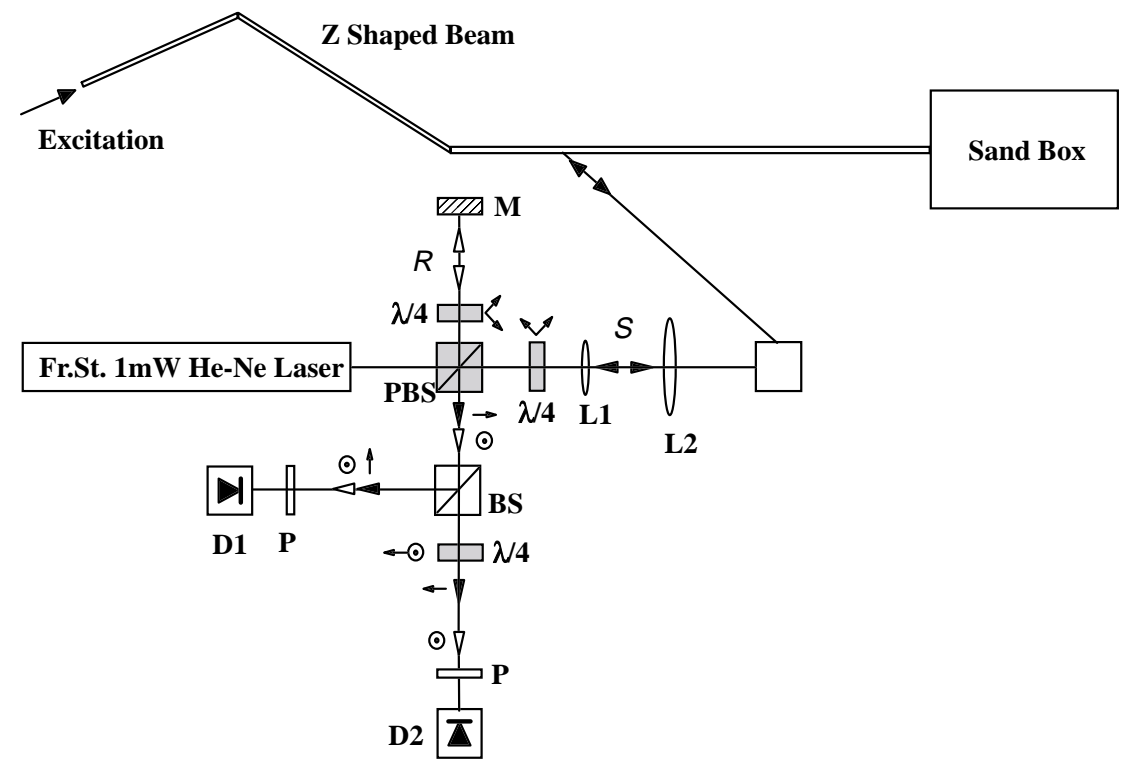

Fig. 2. Simplified scheme of the homodyne LDV.

is mixed with an optical reference beam and projected onto a detector. The detector is a nonlinear device with an output proportional to the power of the incident light, i.e., the square of the optical electric field. Thus, the coherent addition (interference) of the scattered beam collected from the object with the reference beam results in a voltage output proportional to the difference of the two optical frequencies. The frequency $\nu_{D}$ (called Doppler frequency) of this alternate signal can be written as $\nu_{D}=2 u / \lambda$, where $u$ is the component of the velocity in the direction of the incident beam and $\lambda$ is the light wavelength. For He-Ne laser light in vacuum $\lambda=0.6328 \mu \mathrm{m}$ and $\nu_{D} / u \approx 3,16 \mathrm{MHz} / \mathrm{m} / \mathrm{s}$.

One of the simplest optical sensors for a LDV is the Michelson interferometer [2]. In its original configuration (using a fixed mirror in the reference arm), the Michelson interferometer can be employed for measuring vibrations when the displacement of the object is somewhat higher than the laser wavelength and when the knowledge of the direction of the velocity is of no importance. However, most applications require the discrimination of the direction of the velocity, which is impossible by this scheme. In fact, although the Doppler frequency shift depends on the direction of the motion, the output of such an interferometer is symmetric with respect to the sign of velocity. The most commonly used approach to discriminate the direction of the motion is to use a frequency shifting device in the reference laser beam to break the symmetry of the optical output. These are called heterodyned systems $[7$,
12]. In this present experiment, an optical setup based on the scheme used by Jackson et al. [8] was implemented. The homodyned system mixes the Doppler shifted laser beam from the object with a reference beam, which is not frequency shifted, as in the case of the classical Michelson interferometer. The directional ambiguity problem is solved by generating two optical signals in phase quadrature.

A simplified scheme of the optical setup is shown in Fig. 2. The linearly polarized light from a $1 \mathrm{~mW}$ frequency-stabilized He-Ne laser is split into orthogonal linear polarization components by the polarization beam splitter (PBS). The reference beam (R) is circularly polarized by using a quarter wave plate $(\lambda / 4)$. After passing again through the plate, the polarization of the reflected light from the mirror (M) is rotated by $90^{\circ}$ with respect to the polarization of the incoming wave and it is transmitted by the PBS cube. A similar procedure is used in the $\mathrm{S}$ direction, where a beamexpanding telescope is used to focus the laser beam onto the point of interest. A system of two sliding orthogonal mirrors allows easy selection of the measurement point and the angle of incidence. In this way, the light collected from the mirror and the object are transmitted and reflected by the PBS cube, respectively. These two orthogonal linearly polarized beams are then split into two channels by the nonpolarizing beam splitter cube (BS), resulting in two exactly-similar samples of reference and scattered light having the same phase difference in each channel. A 
quarter wave plate is placed in one channel to produce an additional $90^{\circ}$ phase shift between the beams. To obtain interference from these two beams, $45^{\circ}$ components are taken with polarizers $(\mathrm{P})$, and the mixed optical signals are projected onto the photodetectors (D). The alternate terms of the resulting voltages from $D_{1}$ and $D_{2}$ can be written as $V_{X}=V_{0} \cos \left(2 \pi \nu_{D} t+\varphi_{0}\right)$ and $V_{Y}=V_{0} \sin \left(2 \pi \nu_{D} t+\varphi_{0}\right)$, where $\varphi_{0}$ is a constant phase term (neglecting noise effects introduced by thermal fluctuations and mechanical instabilities in the interferometer) and $V_{0}$ is a voltage depending, among other parameters, on the scattered light collected from the object [5].

The LDV used in this work is being developed in a collaboration between the Vibroacoustics Laboratory and the Optics Laboratory of the University of Campinas. The $V_{X}$ and $V_{Y}$ signals are acquired by a digital oscilloscope and numerically processed for displaying velocities, using a procedure similar to that described by Koo et al. [9]. First attempts to make these experiments employed a first-generation heterodyne Doppler vibrometer, which uses a rotating disk to modulate the frequency of the optical reference beam. Although useful and inexpensive, this method of modulation adds random phase and amplitude noise in the resulting Doppler signals. Compared with this first-generation instrument, the results obtained using our home-made vibrometer had superior signal-to-noise ratios. Certainly, the experiments here described could also be performed using any state-of-the-art commercial laser vibrometer. However, the development of an instrument, besides its educational value, potentially allows the implementation of special optical setups which may be necessary in particular applications.

\section{The experiment}

\subsection{Experimental set-up}

An aluminum beam was shaped in such a way that torsional, longitudinal, and flexural waves would develop in the measurement zone indicated in the Fig. 1. The twisted $\mathrm{z}$-shaped beam consists of one straight beam originally $2 \mathrm{~m}$ long which was folded at three different locations along its length so that four straight spans $(\mathrm{AB}, \mathrm{BC}, \mathrm{CD}$, and $\mathrm{DE})$ were formed. Span $\mathrm{AB}$ is parallel to the $x$ axis and is $1.4 \mathrm{~m}$ long, with $0.4 \mathrm{~m}$ plunged in the sand box. Span BC is in the $x y$ plane; it is $0.3 \mathrm{~m}$ long and was produced by a rotation of $39^{\circ}$ in the positive $z$ direction. Span $\mathrm{CD}$ is $0.28 \mathrm{~m}$ long and was generated by folding the beam along a line that makes $45^{\circ}$ with respect to the direction normal to the neutral axis of span BC. The folding consisted of a rotation of $-45^{\circ}$ with respect to the axis defined by the folding line $\left(z^{\prime \prime}\right)$. Finally, in order to have a flat surface where to bond a force transducer, span DE was obtained by folding the beam along the line orthogonal to its neutral axis with a rotation of $90^{\circ}$. The excitation was applied normally to span $\mathrm{DE}$, which corresponds to exciting longitudinally the free end of span CD.

Using different combinations of measurements taken using PVDF patches and LDV, it is possible to separate the contribution of each one of the displacements associated with different types of waves and, therefore, with different SI components. Eight PVDF patches were used in the configuration shown in Fig. 1.

By combining the response measured at each of the PVDFs, it is possible to separate the contributions of the different types of waves. The measured strain distribution can be decomposed into the sum of an anti-symmetric distribution - the bending component - with respect to the center of the beam (e.g., PVDF1PVDF3+PVDF2-PVDF4 on cross-section 1 in Fig. 1) and a uniform strain distribution - the longitudinal component (e.g., PVDF1+PVDF3+PVDF2+PVDF4 on cross-section 1 in Fig. 1). With this PVDF configuration it is not possible to measure the torsional contribution, which is expected to be small in this example, as will be shown with the LDV measurements.

Now, using LDV and measuring at 4 points in 2 different sections of the beam (corresponding to the center of the PVDFs in Fig. 1, at the edges of the beam), aimed at with different angles, it is possible to separate the contribution due to the three different waves: bending, longitudinal and torsional. At each of the points two measurements were taken at $+45^{\circ}$ and at $-45^{\circ}$, on the $x y$-plane, relative to the $y$ direction. The out-of-plane velocity was calculated by adding the measurements taken at each location at $+45^{\circ}$ and $-45^{\circ}$ and dividing the result by $\sqrt{2}$. The in-plane velocity is simply the difference between the two measurements divided by $\sqrt{2}$. The torsional component is obtained by subtracting the out-of-plane components measured at the upper and lower edges of the beam section and dividing the result by the beam width. Thus, the separated velocity components associated with the different kinds of waves are obtained.

\subsection{Experimental results}

In order to apply the techniques described above, the $z$-shaped beam structure was excited by an electrody- 


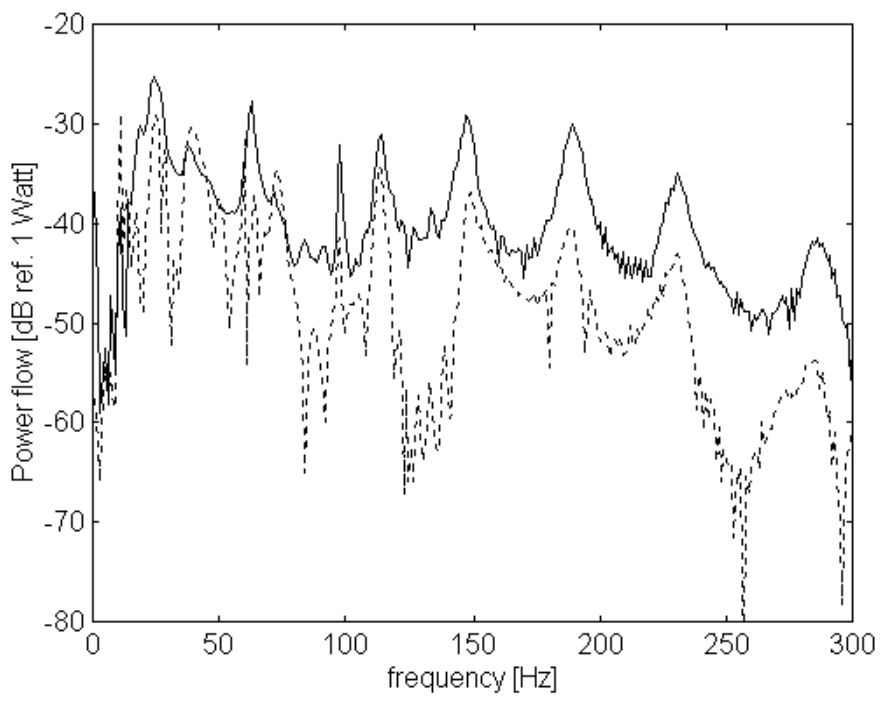

Fig. 3. Input power versus active SI measured with PVDF patches. — Input power; $\cdots$ active SI.

namic shaker using a periodic chirp signal. The period of the chirp was $250 \mathrm{~ms}$ in the case of the LDV test and $1 \mathrm{~s}$ in the case of the PVDF test, corresponding to a frequency resolution of $4 \mathrm{~Hz}$ and $1 \mathrm{~Hz}$, respectively. The time length has to be restricted to accommodate the LDV signal processing requirements. Due to the fact that the LDV signal processing was done off-line, with digitized data, there was a compromise between the high sampling rate necessary to resolve the high frequency Doppler signal, and a sufficient signal length for the resolution of the low frequency velocity signal. 10 blocks of 15000 samples each were acquired at a rate of $50 \mathrm{kHz}$. Also, because of this limitation (which can be overcome using an analog signal processing board to extract the velocity signal from the photo-detector signals), the magnitude of the excitation force was adjusted so that the maximum Doppler shift was approximately $3 \mathrm{kHz}$ (corresponding to a maximum velocity of about $10^{-3} \mathrm{~m} / \mathrm{s}$ ). Therefore, the active power input fed to the structure by the shaker was much smaller in the LDV test than in the PVDF tests.

In order to improve the signal-to-noise ratio, Frequency Response Functions between the force transducer signal and the LDV or PVDF signals were calculated. Structural intensity spectra were computed using the cross spectra between the input force and each measured signal, which is the FRF multiplied by the auto power spectrum of the input force.

Figure 3 shows a comparison of the input power and the total structural intensity computed from measurements made with PVDFs. It can be observed that at higher frequencies the computed SI is smaller than the input power. This can be explained partly by the influence of the PVDFs which, together with their cabling, absorb part of the incoming energy. Figure 4 shows the relative contributions of the bending and longitudinal waves to the total intensity. It can be observed that, at these test force levels, the longitudinal waves carry more active power than the flexural waves.

Figure 5 shows the results obtained with LDV measurements (with the PVDF patches and associated cabling removed). There is a better agreement between input power and total structural intensity. The fact that the total SI does not decrease with frequency, can be explained by the fact that the LDV is non-intrusive and thus, the energy flow is preserved. Figure 6 shows the relative contributions of the different waves to the total SI. It can be observed that, in this test, with much smaller power levels (compare the magnitudes with Fig. 4), the flexural and longitudinal waves have contributions of similar magnitudes to the total SI. This can be explained by the different behavior of the sand box at different vibration levels. At lower levels most of the energy is dissipated through flexural motion, while at higher amplitude levels, most of the dissipation is due to longitudinal motion. To verify this hypothesis, another test was carried out at an intermediate force level. Results in Fig. 7 confirm that, for lower force levels, the contribution of the longitudinal waves decreases with respect to that of the flexural waves. 


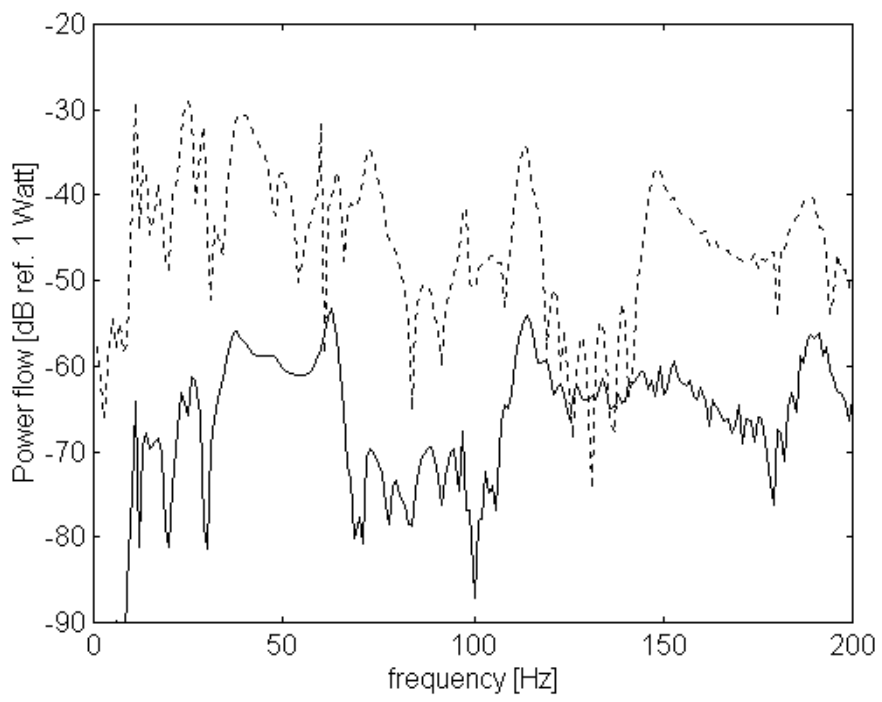

Fig. 4. SI components measured using PVDF patches. - Flexural SI; $\cdots$ longitudinal SI.

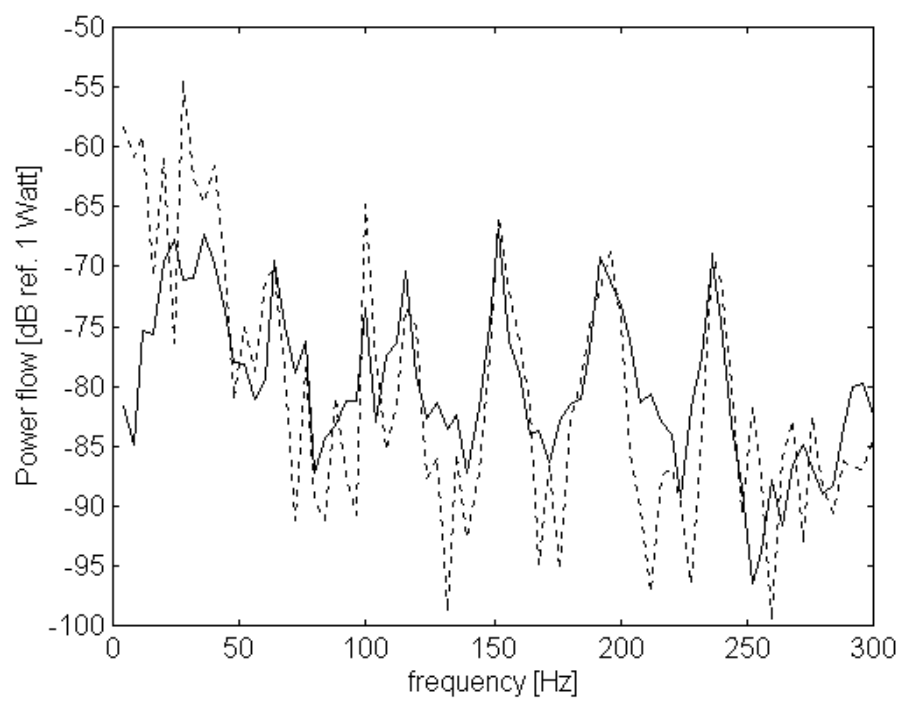

Fig. 5. Input power versus active SI - LDV. - Input power; $\cdots$ active SI.

The torsional contribution, as expected (due to the geometry of the $z$-shaped beam), is much smaller than the other two components.

\section{Conclusions}

Structural intensity measurements are difficult to carry out because the active part of the total vibration energy is usually very small compared with the reac- tive part. Furthermore, conventional instrumentation may alter the structural intensity pattern by absorbing energy locally. In this paper, the formulation of the structural intensity propagated via the three types of waves in beams (flexural, longitudinal, and torsional) was reviewed. Sensor placement and signal combinations that separate the three types of waves were described. Piezoelectric film sensor (PVDF) and LDV measurements were used.

A $z$-shaped beam was used to investigate the pro- 


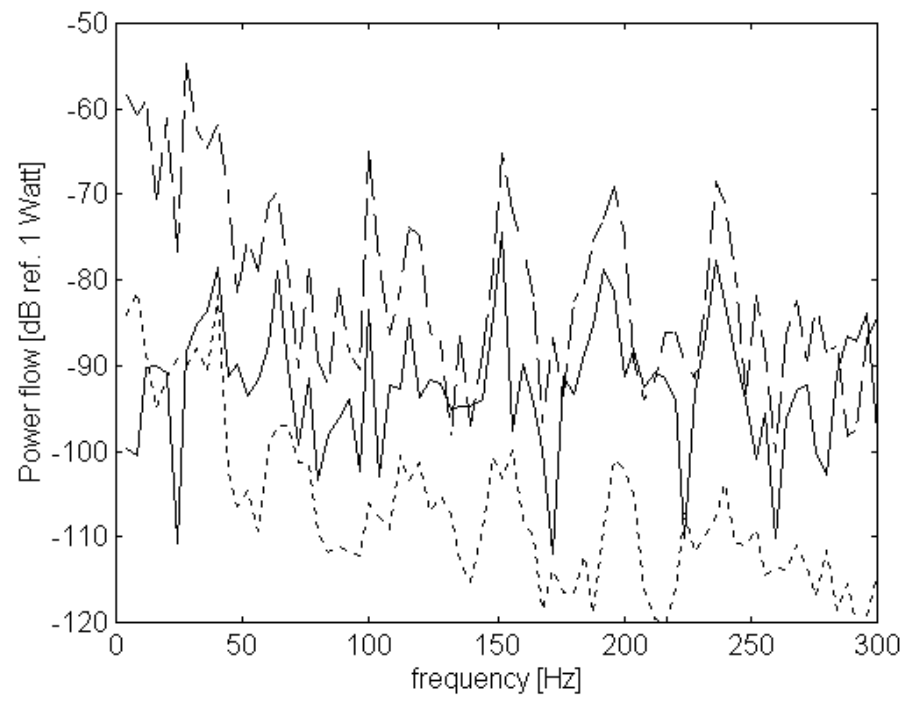

Fig. 6. SI components measured using the LDV. - - - Flexural SI; — longitudinal SI; - - torsional SI.

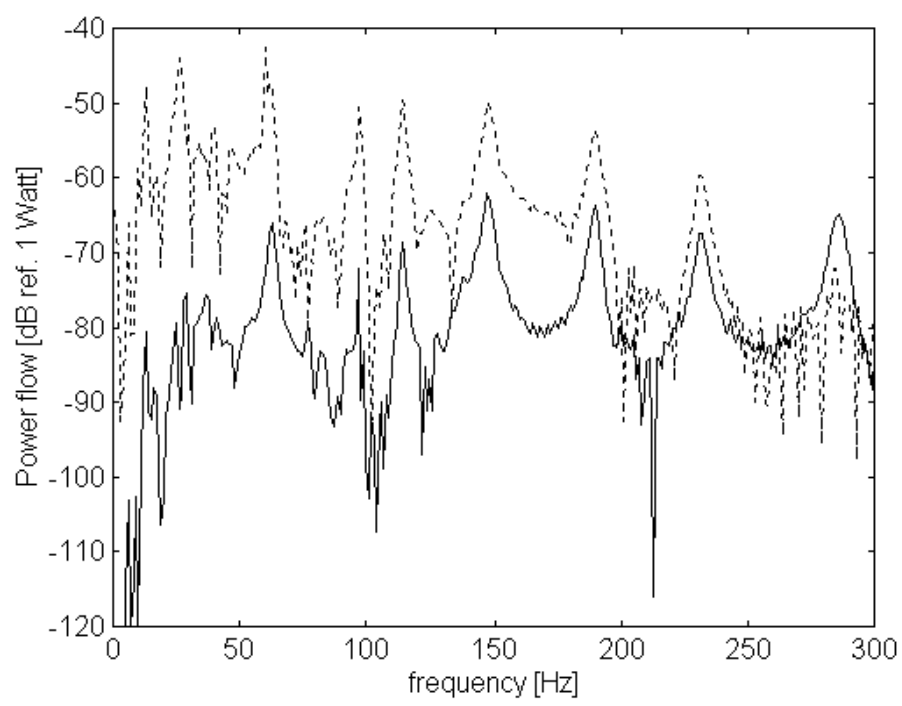

Fig. 7. SI components at a lower vibration level measured using PVDF patches. — Flexural SI; $\cdots$ longitudinal SI.

posed techniques experimentally. With the LDV, the three types of waves were measured, while with the PVDF arrangement, only the longitudinal and flexural waves were obtained. It was shown that, in the frequency range investigated $(\mathrm{DC}-300 \mathrm{~Hz})$, the torsional wave contribution was very small.

The wave component approach was used to estimate the structural intensity components. It is more accurate than the finite difference because it does not require approximation of the spatial derivatives necessary to evaluate the structural intensity. Besides, it is straightforward to obtain one expression from the other. The finite difference approximation is obtained simply by replacing " $\sin (k \Delta x)$ " by " $k \Delta x$ " in the denominator of the expression obtained using the wave component approach. When using the wave component, attention must be paid to the frequency range under investigation, as the function $\sin (k \Delta x)$ appears in the denominator and may cause singularity in the intensity estimation.

Besides the wave component approach, the far-field 


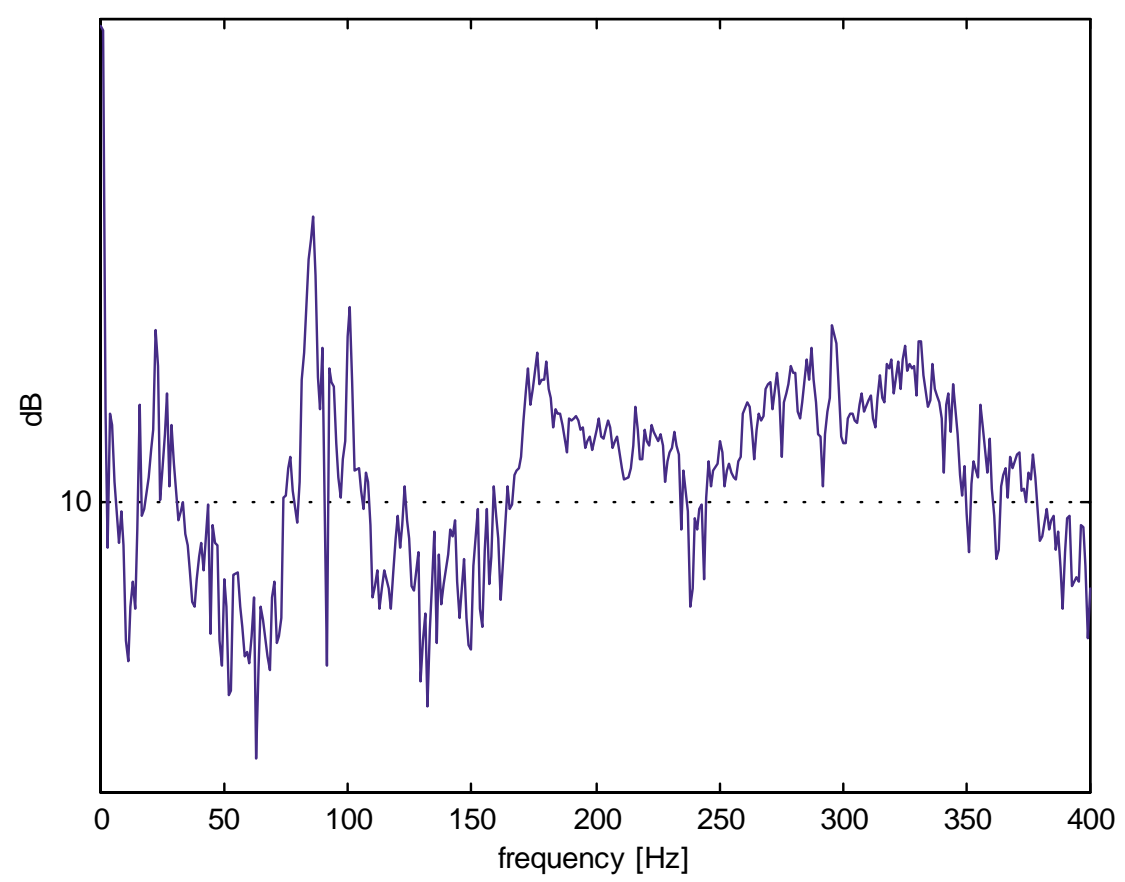

Fig. 8. Ratio between in-plane component to the $2^{\circ}$ angle error component.

approximation was assumed in the evaluation of the structural intensity components. Some authors [14] state that for calculating the flexural component the farfield consideration is valid when $d>\lambda_{f} / 2$, where $d$ is the distance between the excitation and the measurement points and $\lambda_{f}$ is the flexural wavelength at the excitation frequency. Therefore, in real structures, in order to make the far-field assumption, attention has to be paid to the excitation frequency range and to the distance between the measurement point and any discontinuities.

The LDV used in this work, developed in our laboratories, was briefly described. Due to the fact that the signal processing of the photo-detector signals was done numerically and off-line, there was a compromise between the sampling frequency, necessary to acquire the high frequency Doppler carrier frequency and the low frequency modulation. Thus, in the tests performed, using LDV, the vibration levels had to be considerably lower than in the tests performed using PVDF sensors. This limitation will be overcome with the implementation of an analog signal processing circuit.

In order to verify the sensitivity of the technique presented here with respect to angle errors in the laser measurements, an error of $2^{\circ}$ was considered and the in-plane component variation associated with this error was evaluated. Figure 8 shows the ratio between in-plane component and the $2^{\circ}$ angle error component.
It was considered that for ratios larger than 3 the measurements were meaningful. In the $z$-shaped beam investigated here this ratio happens above $170 \mathrm{~Hz}$.

It was observed that, at higher vibration levels, with this particular set up, the longitudinal waves were responsible for the propagation of most of the input energy, while at lower vibration levels, the flexural waves were as effective as the longitudinal waves in conveying the energy. A test at an intermediate vibration level confirmed this hypothesis. This tendency is presumably linked to the behavior of the imperfect anechoic termination used in the experiments (sand box).

\section{Acknowledgments}

The authors wish to acknowledge the Brazilian research funding agency CNPq (Proc. No. 620705/949) and FAPESP (Proc. No. 97/00511-0 and Proc. No. 98/15720-7) for their financial support.

\section{References}

[1] P.D. Bauman, Measurement of Structural intensity: analytic and experimental evaluation of various techniques for the case of flexural waves in one-dimensional structures, Journal of Sound and Vibration 174(5) (1994), 677-694. 
[2] R.H. Belansky and K.H. Wanser, Laser Doppler velocimetry using a bulk optic Michelson interferometer: a student laboratory experiment, American journal of physics 61(11) (1993), 1014-1019.

[3] Y.H. Berthelot, M. Yang and J. Jarzinski, Recent progress on laser Doppler measurements in structural acoustics, in: Proceedings of the 4th International Congress on Intensity Techniques, Senlis, France, 1993, pp. 199-206.

[4] L. Cremer, M. Heckl and E.E. Ungar, Structure-Borne Sound, Springer, Berlin, 1988 .

[5] J.W. Goodman, Some fundamental properties of speckles, Journal of the optical society of America 66(11) (1976), 11451150.

[6] C.R. Halkyard and B.R. Mace, Wave Component Approach to Structural Intensity in Beams, in: Proceedings of the 4th International Congress on Intensity Techniques, Senlis, France, 1993, pp. 183-190.

[7] N.A. Halliwell, Laser Doppler measurement of vibrating surfaces: a portable instrument, Journal of Sound and Vibration 62(2) (1979), 312-315.

[8] D.A. Jackson, A.D. Kersey and A.C. Lewin, Fibre Gyroscope with passive quadrature detection, Electronics Letters 20(10) (1984), 399-401.

[9] K.P. Koo, A.B. Tveten and A. Dandridge, Passive stabilization scheme for fiber interferometers using $(3 \times 3)$ fiber directional couplers, Appl. Phys. Lett. 41(7) (1982), 616-618.

[10] D.U. Noiseux, Measurement of power flow in uniform beams and plates, Journal of the acoustical society of America 47 (1970), 238-247.

[11] G. Pavic, Measurement of structure borne wave intensity, part I: formulation of methods, Journal of Sound and Vibration 49(2) (1976), 221-230.

[12] T.A. Riener, A.C. Goding and F.E. Talke, Measurement of head/disk spacing modulation using a two channel fiber optic laser Doppler vibrometer, IEEE Transactions on Magnetics 24(6) (1988), 2745-2747.

[13] J.W. Verheij, Cross-spectral density methods for measuring structure-borne power flow on beams and pipes, Journal of Sound and Vibration 70 (1980), 133-139.

[14] G.P. Gibbs, Simultaneous active control of flexural and extensional power flow in thin beams, Ph.D. thesis, Virginia Polytechnic Institute and State University, USA, 1995. 

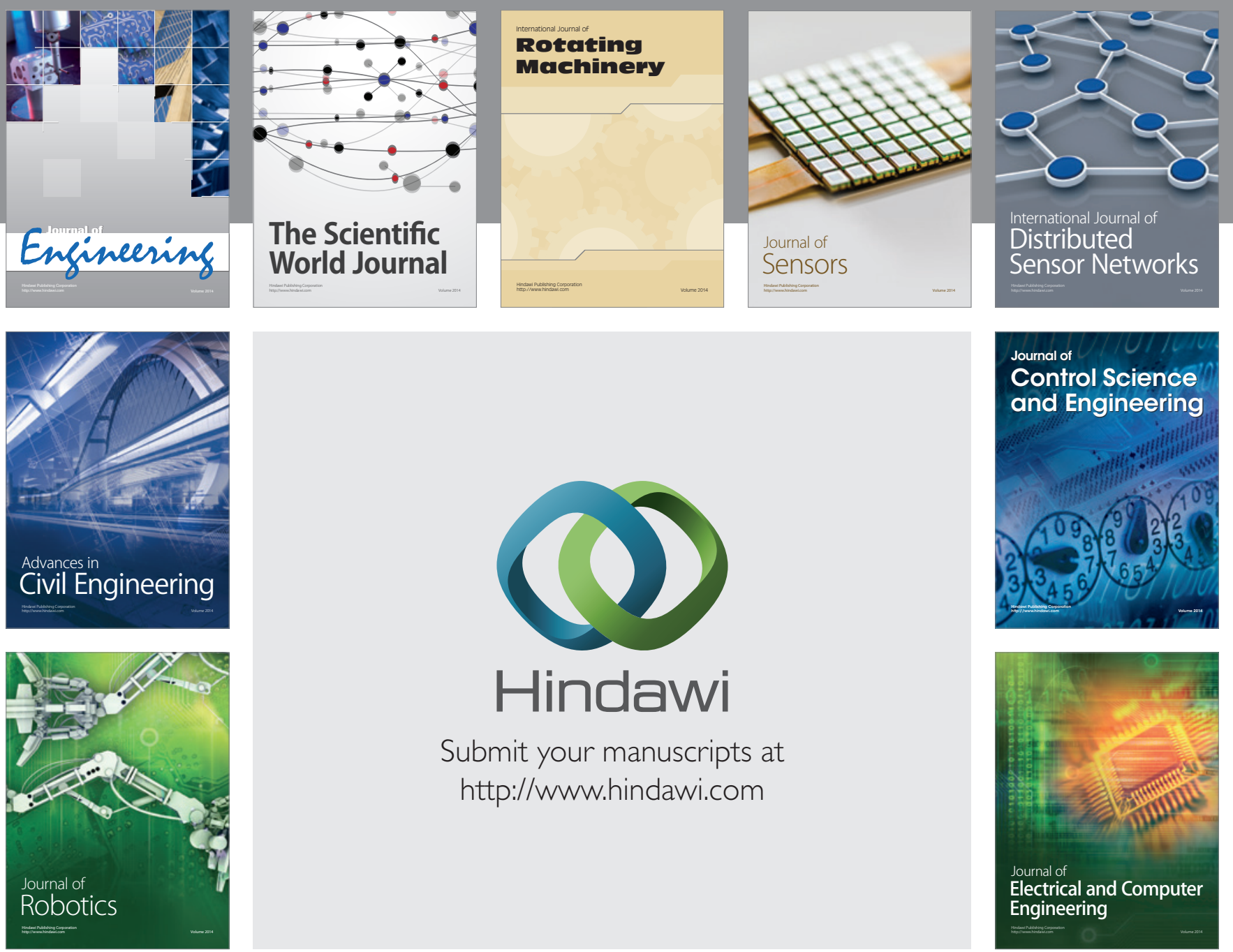

Submit your manuscripts at

http://www.hindawi.com
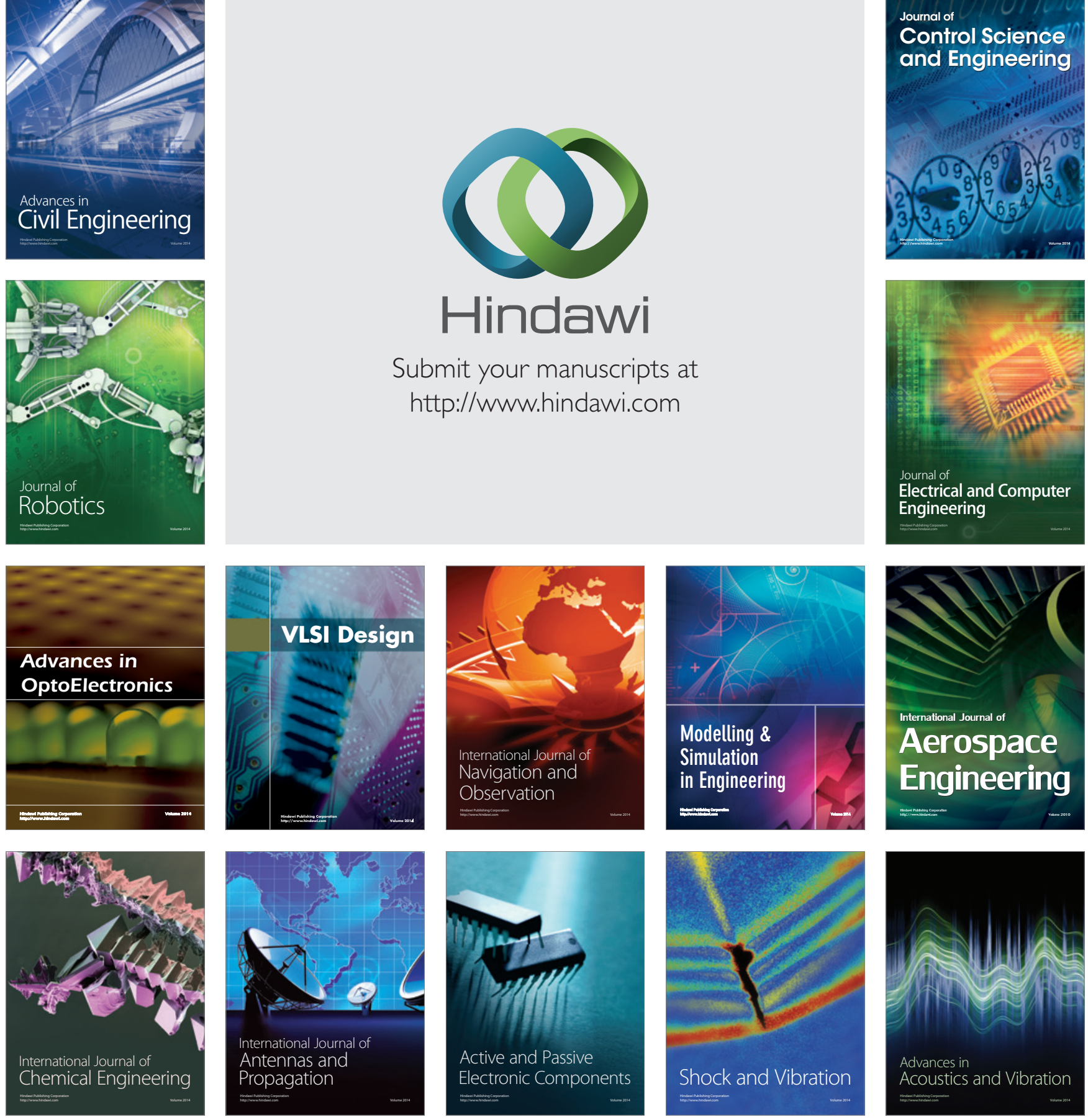